\title{
Complete genome sequence of Beutenbergia cavernae type strain (HKI 0122 ${ }^{\mathrm{T}}$ )
}

\author{
Miriam Land ${ }^{1,2}$, Rüdiger Pukall ${ }^{3}$, Birte Abt ${ }^{3}$, Markus Göker ${ }^{3}$, Manfred Rohde ${ }^{4}$, Tijana Glavina \\ Del Rio" ${ }^{1}$, Hope Tice ${ }^{1}$, Alex Copeland", Jan-Fang Cheng ${ }^{1}$, Susan Lucas ${ }^{1}$, Feng Chen ${ }^{1}$, Matt No- \\ lan', David Bruce ${ }^{1,5}$, Lynne Goodwin ${ }^{1,5}$, Sam Pitluck ${ }^{1}$ Natalia Ivanova ${ }^{1}$, Konstantinos Ma- \\ vromatis ${ }^{1}$, Galina Ovchinnikova ${ }^{1}$, Amrita Pati ${ }^{1}$, Amy Chen ${ }^{6}$, Krishna Palaniappan ${ }^{6}$, Loren \\ Hauser $^{1,2}$, Yun-Juan Chang ${ }^{1,2}$, Cynthia C. Jefferies ${ }^{1,2}$, Elizabeth Saunders ${ }^{5}$, Thomas Brettin ${ }^{1,5}$, \\ John C. Detter ${ }^{1,5}$, Cliff Han ${ }^{1,5}$, Patrick Chain ${ }^{1,7}$, James Bristow ${ }^{1}$, Jonathan A. Eisen ${ }^{1,8}$, Victor \\ Markowitz ${ }^{6}$, Philip Hugenholtz ${ }^{1}$, Nikos C. Kyrpides ${ }^{1}$, Hans-Peter Klenk ${ }^{3}$, and Alla Lapidus ${ }^{{ }^{*}}$ \\ ${ }^{1}$ DOE Joint Genome Institute, Walnut Creek, California, USA \\ ${ }^{2}$ Oak Ridge National Laboratory, Oak Ridge, Tennessee, USA \\ ${ }^{3}$ DSMZ - German Collection of Microorganisms and Cell Cultures GmbH, Braunschweig, \\ Germany \\ ${ }^{4} \mathrm{HZI}$ - Helmholtz Centre for Infection Research, Braunschweig, Germany \\ ${ }^{5}$ Los Alamos National Laboratory, Bioscience Division, Los Alamos, New Mexico USA \\ ${ }^{6}$ Biological Data Management and Technology Center, Lawrence Berkeley National Labora- \\ tory, Berkeley, California, USA \\ ${ }^{7}$ Lawrence Livermore National Laboratory, Livermore, California, USA \\ ${ }^{8}$ University of California Davis Genome Center, Davis, California, USA
}

*Corresponding author: $\underline{\text { Alla Lapidus }}$

Keywords: mesophile, non-pathogenic, aerobic and microaerophilic, rod-coccus growth cycle, MK-8( $\left.\mathrm{H}_{4}\right)$, actinomycete, Micrococcineae

Beutenbergia cavernae (Groth et al. 1999) is the type species of the genus and is of phylogenetic interest because of its isolated location in the actinobacterial suborder Micrococcineae. $B$. cavernae $\mathrm{HKI} 0122^{\top}$ is a Gram-positive, non-motile, non-spore-forming bacterium isolated from a cave in Guangxi (China). B. cavernae grows best under aerobic conditions and shows a rod-coccus growth cycle. Its cell wall peptidoglycan contains the diagnostic L-lysine $\leftarrow$ Lglutamate interpeptide bridge. Here we describe the features of this organism, together with the complete genome sequence, and annotation. This is the first completed genome sequence from the poorly populated micrococcineal family Beutenbergiaceae, and this 4,669,183 bp long single replicon genome with its 4225 protein-coding and 53 RNA genes is part of the Genomic Encyclopedia of Bacteria and Archaea project.

\section{Introduction}

Beutenbergia cavernae strain HKI 0122 ${ }^{\mathrm{T}}$ (DSM $12333=$ ATCC BAA-8 = JCM 11478) is the type strain of the species, which represents the type species of the genus Beutenbergia, the type genus of the family Beutenbergiaceae [1]. B. cavernae was described by Groth et al. 1999 as Grampositive, non-motile and non-spore-forming [1].
The organism is of significant interest for its position in the tree of life within the small (2 type strains) family Beutenbergiaceae Zhi, et al., 2009 emend. Schumann et al. 2009 in the actinobacterial suborder Micrococcineae [2], which in addition to the genus Beutenbergia contains only the genus Salana $[3,4]$ (Figure 1), also otherwise stated in a 
recent overview on the class Actinobacteria [2]. Here we present a summary classification and a set of features for B. cavernae strain HKI $0122^{\mathrm{T}}$ (Table 1), together with the description of the complete genome sequencing and annotation.

In addition to strain HKI $0122^{\mathrm{T}}$, only one other strain (HKI 0132) was isolated from the soil sample collected in the Reed Flute Cave near Guilin, Guangxi, China. HKI 0132 was also classified in the species $B$. cavernae [1]. No closely related isolates and uncultivated clones with more than $97 \% 16 \mathrm{~S}$ rRNA gene sequence identity are recorded in the microbiological literature, nor can any phylotype from environmental samples or genomic surveys be directly linked to $B$. cavernae.

$B$. cavernae cells vary in shape and colonies grown on rich medium vary in color from cream to bright yellow. In young cultures, cells are irregular rods arranged in palisades, clusters or in pairs at an angle to give $\mathrm{V}$-formations [1]. Cells in stationary cultures are predominantly coccoid, occurring singly, in pairs, irregular clusters and short chains. During growth in complex media a rod-coccus growth cycle was observed [1]. B. cavernae grows well under aerobic and microaerophilic conditions, but not under anaerobic conditions [1]. The optimal growth temperature is $28^{\circ} \mathrm{C}$ [1].

B. cavernae is able to degrade casein, esculin, gelatin and potato starch. Acids are produced from Larabinose, D-cellobiose, dextrin, D-fructose, Dgalactose, D-glucose, glycerol, inulin, maltose, Dmannose, D-raffinose, L-rhamnose, D-ribose, salicin, sucrose, starch, trehalose and D-xylose. There is no acid production from D-glucitol, lactose and D-mannitol. Nitrate is reduced to nitrite, $\mathrm{H}_{2} \mathrm{~S}$ is produced [1].

\section{Classification and features}

Figure 1. shows the phylogenetic neighborhood of B. cavernae strain HKI $0122^{\mathrm{T}}$ in a $16 \mathrm{~S}$ rRNA based tree. Analysis of the two identical 16S rRNA gene sequences in the genome of strain HKI differed by four nucleotides from the previously published 16S rRNA sequence generated from DSM 12333 (Y18378). The slight differences between the genome data and the reported 16S rRNA gene sequence is most likely due to sequencing errors in the previously reported sequence data .

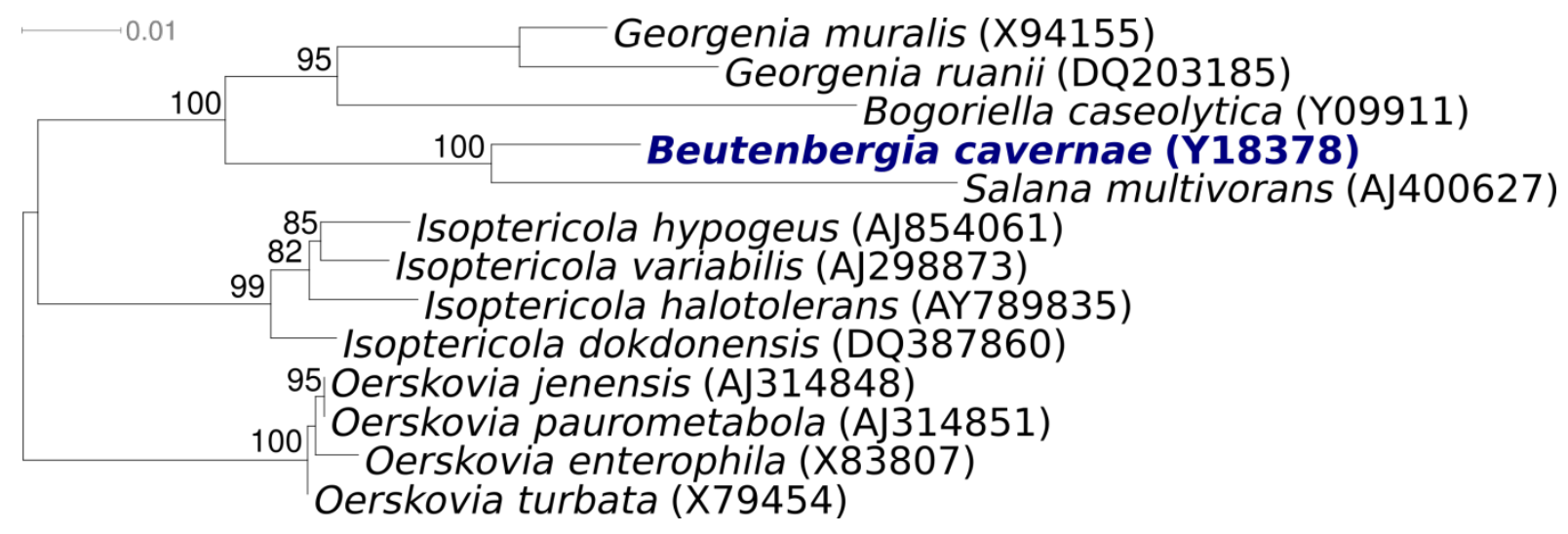

Figure 1. Phylogenetic tree of $B$. cavernae HKI $0122^{\top}$ and all type strains of the genus Beutenbergia, inferred from 1,411 aligned characters $[5,6]$ of the $16 \mathrm{~S}$ rRNA sequence under the maximum likelihood criterion [7]. The tree was rooted with species from the genera Isoptericola and Oerskovia, both also members of the actinobacterial suborder Micrococcineae. The branches are scaled in terms of the expected number of substitutions per site. Numbers above branches are support values from 1,000 bootstrap replicates if larger than $60 \%$. Strains with a genome-sequencing project registered in GOLD [8] are printed in blue; published genomes in bold. 


\section{Chemotaxonomy}

The peptidoglycan of B. cavernae HKI $0122^{\mathrm{T}}$ contains D- and L-alanine, D- and L-glutamic acid and L-lysine, with the latter widely distributed among actinobacteria [1]. The strain possesses a type A4< peptidoglycan with a diagnostic L-Lys $\leftarrow$ L-Glu interpeptide bridge, type A11.54 according to DSMZ. Glucose, mannose and galactose are the cell wall sugars [1]. The fatty acid profile of strain B. cavernae HKI $0122^{\mathrm{T}}$ is dominated by 13 -methyl tetradecanoic (iso- $\mathrm{C}_{15: 0} ; 43.7 \%$ ) and 12-methyl tetradecanoic (anteiso- $\mathrm{C}_{15: 0} ; 34.6 \%$ ) saturated, branched chain acids. Other predominantly saturated fatty acids play a minor role in the cellular fatty acid composition of the strain: iso- $\mathrm{C}_{14: 0}(0.9 \%), \mathrm{C}_{14: 0}$
(1.9\%); $\mathrm{C}_{15: 0}(0.9 \%)$ iso $\mathrm{C}_{16: 0}(2.3 \%), \mathrm{C}_{16: 0}(6.8 \%)$, isoC $_{17: 0}(3.1 \%)$, anteiso- $\mathrm{C}_{17: 0}(4.9 \%)$, und $\mathrm{C}_{18: 1}$ $(0.9 \%)$ [1]. Mycolic acids are not present [1]. MK$8\left(\mathrm{H}_{4}\right)$ is the major menaquinone, complemented by minor amounts of $\mathrm{MK}-8\left(\mathrm{H}_{2}\right), \mathrm{MK}-8$ and $\mathrm{MK}-$ $9\left(\mathrm{H}_{4}\right)$ [1]. The combination of the L-Lys $\leftarrow$ L-Glu interpeptide bridge and MK-8 $\left(\mathrm{H}_{4}\right)$ as the dominating menaquinone is shared with the organisms from the neighboring genera Bogoriella and Georgenia. The polar lipids of strain HKI $0122^{\mathrm{T}}$ consist of phosphatidylinositol and diphosphatidylglycerol together with three yet unidentified phospholipids [1].

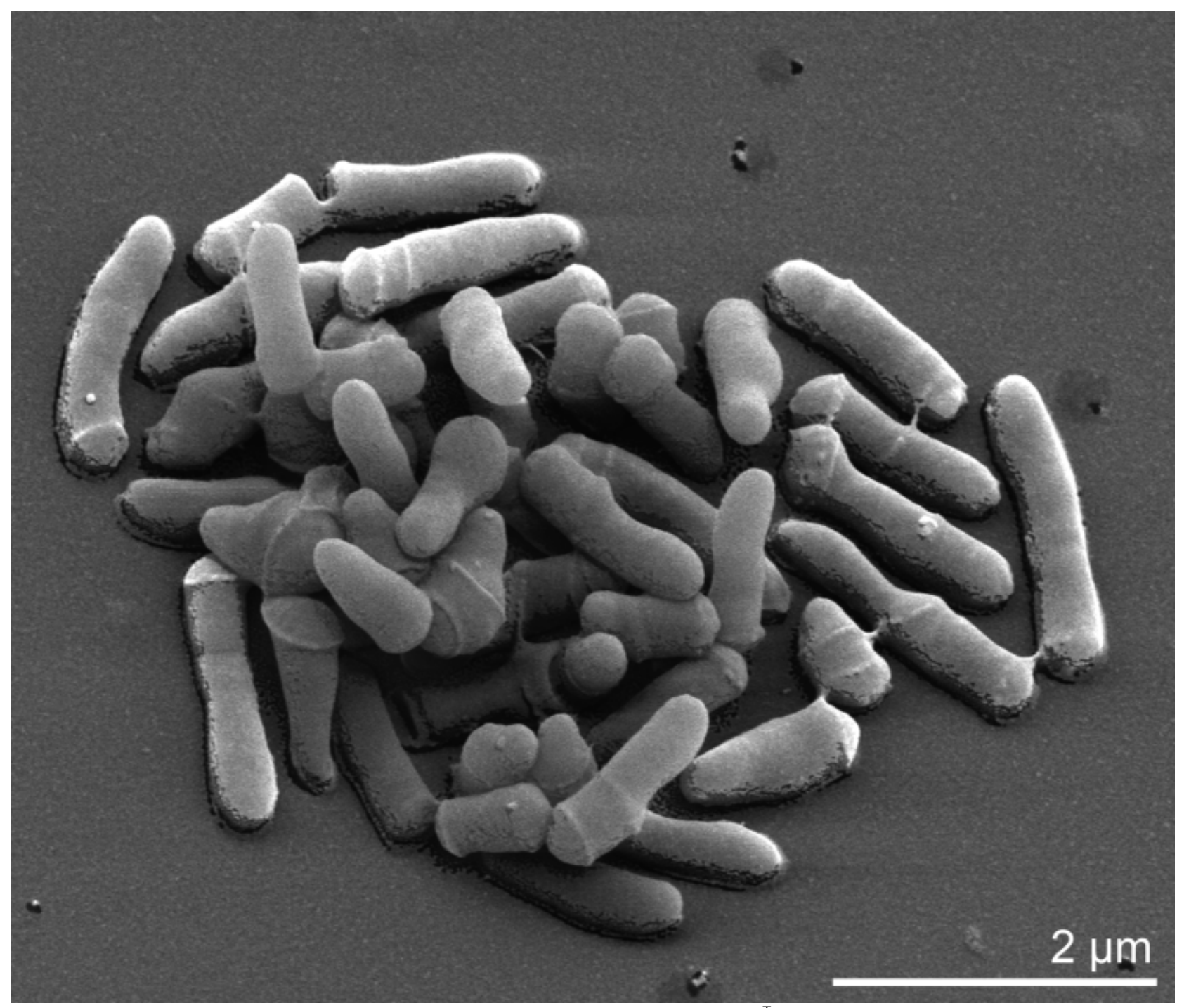

Figure 2. Scanning electron micrograph of B. cavernae HKI $0122^{\top}$ 
Table 1. Classification and general features of B. cavernae HKI $0122^{\top}$ based on the MIGS recommendations [9]

\begin{tabular}{|c|c|c|c|}
\hline MIGS ID & Property & Term & $\begin{array}{l}\text { Evidence } \\
\text { code }\end{array}$ \\
\hline & \multirow{9}{*}{ Current classification } & Domain Bacteria & \\
\hline & & Phylum Actinobacteria & \\
\hline & & Class Actinobacteria & TAS [10] \\
\hline & & Order Actinomycetales & TAS [10] \\
\hline & & Suborder Micrococcineae & TAS [2] \\
\hline & & Family Beutenbergiaceae & TAS [2] \\
\hline & & Genus Beutenbergia & TAS [1] \\
\hline & & Species Beutenbergia cavernae & TAS [1] \\
\hline & & Type strain HKI 0122 & \\
\hline & Gram stain & positive & TAS [1] \\
\hline & Cell shape & varies; rod-coccus growth cycle & TAS [1] \\
\hline & Motility & nonmotile & TAS [1] \\
\hline & Sporulation & non-sporulating & TAS [1] \\
\hline & Temperature range & mesophile & TAS [1] \\
\hline & Optimum temperature & $28^{\circ} \mathrm{C}$ & TAS [1] \\
\hline & Salinity & tolerance of $2-4 \%(\mathrm{w} / \mathrm{v}) \mathrm{NaCl}$ & TAS [1] \\
\hline \multirow[t]{3}{*}{ MIGS-22 } & Oxygen requirement & $\begin{array}{l}\text { aerobic and microaerobic, no growth under anaerobic } \\
\text { conditions }\end{array}$ & TAS [1] \\
\hline & Carbon source & glucose, maltose, mannose, cellobiose & TAS [1] \\
\hline & Energy source & unknown & \\
\hline MIGS-6 & Habitat & cave (soil) & TAS [1] \\
\hline MIGS-15 & Biotic relationship & & \\
\hline \multirow[t]{3}{*}{ MIGS-14 } & Pathogenicity & none & NAS \\
\hline & Biosafety level & 1 & TAS [11] \\
\hline & Isolation & cave, soil between rocks & TAS [1] \\
\hline MIGS-4 & Geographic location & Guangxi, China & TAS [1] \\
\hline MIGS-5 & Sample collection time & about 1999 & TAS [1] \\
\hline $\begin{array}{l}\text { MIGS-4.1 } \\
\text { MIGS-4.2 }\end{array}$ & Longitude - Latitude & $110.263306-25.307878$ & TAS [1] \\
\hline MIGS-4.3 & Depth & not reported & \\
\hline MIGS-4.4 & Altitude & not reported & \\
\hline
\end{tabular}

Evidence codes - IDA: Inferred from Direct Assay (first time in publication); TAS: Traceable Author Statement (i.e., a direct report exists in the literature); NAS: Non-traceable Author Statement (i.e., not directly observed for the living, isolated sample, but based on a generally accepted property for the species, or anecdotal evidence). These evidence codes are from the Gene Ontology project [12]. If the evidence code is IDA the property was directly observed for a live isolate by one of the authors or an expert mentioned in the acknowledgements.

\section{Genome sequencing and annotation Genome project history}

This organism was selected for sequencing on the basis of its phylogenetic position, and is part of the Genomic Encyclopedia of Bacteria and Archaea project. The genome project is deposited in the Genomes OnLine Database [8] and the complete genome sequence in GenBank (CP001618). Sequencing, finishing and annotation were performed by the DOE Joint Genome Institute (JGI). A summary of the project information is shown in Table 2. 
Table 2. Genome sequencing project information

\begin{tabular}{|c|c|c|}
\hline MIGS ID & Property & Term \\
\hline MIGS-31 & Finishing quality & Finished \\
\hline MIGS-28 & Libraries used & $\begin{array}{l}\text { Three genomic libraries: two Sanger libra- } \\
\text { ries - } 8 \text { kb pMCL200 and fosmid pcc1Fos - } \\
\text { and } \\
\text { one } 454 \text { pyrosequence standard library }\end{array}$ \\
\hline MIGS-29 & Sequencing platforms & ABI3730, 454 GS FLX \\
\hline MIGS-31.2 & Sequencing coverage & 8.56x Sanger; 10.86x pyrosequence \\
\hline MIGS-30 & Assemblers & Newbler version 1.1.02.15, phrap \\
\hline \multirow[t]{6}{*}{ MIGS-32 } & Gene calling method & Prodigal \\
\hline & INSDC / Genbank ID & СР001618 \\
\hline & Genbank Date of Release & 07-MAY-2009 \\
\hline & GOLD ID & Gc01025 \\
\hline & NCBI project ID & 20827 \\
\hline & Database: IMG-GEBA & 2501416922 \\
\hline \multirow[t]{2}{*}{ MIGS-13 } & Source material identifier & DSM 12333 \\
\hline & Project relevance & Tree of Life, GEBA \\
\hline
\end{tabular}

\section{Growth conditions and DNA isolation}

B. cavernae HKI 0122 ${ }^{\mathrm{T}}$, DSM 12333, was grown in DSMZ medium 736 (Rich Medium) [13] at $28^{\circ} \mathrm{C}$. DNA was isolated from $0.5-1 \mathrm{~g}$ of cell paste using Qiagen Genomic 500 DNA Kit (Qiagen, Hilden, Germany) with a modification of the standard protocol for cell lysis in first freezing for $20 \mathrm{~min}$. $\left(-70^{\circ} \mathrm{C}\right)$, then heating $5 \mathrm{~min} .\left(98^{\circ} \mathrm{C}\right)$, and cooling $15 \mathrm{~min}$ to $37^{\circ} \mathrm{C}$; adding $1.5 \mathrm{ml}$ lysozyme (standard: $0.3 \mathrm{ml}$, only), $1.0 \mathrm{ml}$ achromopeptidase, $0.12 \mathrm{ml}$ lysostaphine, $0.12 \mathrm{ml}$ mutanolysine, 1.5 $\mathrm{ml}$ proteinase $\mathrm{K}$ (standard: $0.5 \mathrm{ml}$, only), followed by overnight incubation at $35^{\circ} \mathrm{C}$.

\section{Genome sequencing and assembly}

The genome was sequenced using a combination of Sanger and 454 sequencing platforms. All general aspects of library construction and sequencing performed at the JGI can be found at the JGI website. 454 Pyrosequencing reads were assembled using the Newbler assembler version 1.1.02.15 (Roche). Large Newbler contigs were broken into 5,256 overlapping fragments of $1,000 \mathrm{bp}$ and entered into the assembly as pseudo-reads. The sequences were assigned quality scores based on Newbler consensus q-scores with modifications to account for overlap redundancy and to adjust inflated q-scores. A hybrid 454/Sanger assembly was made using the parallel phrap assembler (High Performance Software, LLC). Possible mis-assemblies were corrected with Dupfinisher or transposon bombing of bridging clones [14]. Gaps between contigs were closed by editing in Consed, custom primer walking or PCR amplification. A total of 1,627 Sanger finishing reads were produced to close gaps, to resolve repetitive regions, and to raise the quality of the finished sequence. The error rate of the completed genome sequence is less than 1 in 100,000 . Together all sequence types provided 19.42x coverage of the genome.

\section{Genome annotation}

Genes were identified using Prodigal [15] as part of the Oak Ridge National Laboratory genome annotation pipeline, followed by a round of manual curation using the JGIGenePRIMP pipeline [16]. The predicted CDSs were translated and used to search the National Center for Biotechnology Information (NCBI) nonredundant database, UniProt, TIGRFam, Pfam, PRIAM, KEGG, COG, and InterPro databases. Additional gene prediction analysis and functional annotation was performed within the Integrated Microbial Genomes (IMG-ER) platform [17].

\section{Genome properties}

The genome is 4,669,183 bp long and comprises one main circular chromosome with a $73.1 \%$ GC content. (Table 3 and Figure 3). Of the 4,278 genes predicted, 4,225 were protein coding genes, and 53 RNAs. Twenty eight pseudogenes were also identified. The majority of the genes 
(74.3\%) were assigned a putative function while the remaining ones were annotated as hypotheti- cal proteins. The distribution of genes into COGs functional categories is presented in Table 4.

Table 3. Genome Statistics

\begin{tabular}{lrr}
\hline Attribute & \multicolumn{1}{c}{ Value } & \% of Total \\
\hline Genome size (bp) & $4,669,183$ & \\
DNA Coding region (bp) & $4,347,731$ & $93.12 \%$ \\
DNA G+C content (bp) & $3,413,947$ & $73.12 \%$ \\
Number of replicons & 1 & \\
Extrachromosomal elements & 0 & \\
Total genes & 4278 & $100.00 \%$ \\
RNA genes & 53 & $1.24 \%$ \\
rRNA operons & 2 & \\
Protein-coding genes & 4225 & $98.76 \%$ \\
Pseudo genes & 28 & $0.65 \%$ \\
Genes with function prediction & 3183 & $74.40 \%$ \\
Genes in paralog clusters & 689 & $16.11 \%$ \\
Genes assigned to COGs & 3109 & $72.67 \%$ \\
Genes assigned Pfam domains & 3246 & $75.88 \%$ \\
Genes with signal peptides & 1034 & $24.17 \%$ \\
Genes with transmembrane helices & 1135 & $26.53 \%$ \\
CRISPR repeats & 1 & \\
\hline
\end{tabular}

Table 4. Number of genes associated with the 21 general COG functional categories

\begin{tabular}{lrrl}
\hline Code & Value & \multicolumn{1}{c}{$\%$} & Description \\
\hline J & 169 & 4 & Translation, ribosomal structure and biogenesis \\
A & 4 & 0.1 & RNA processing and modification \\
K & 384 & 9.1 & Transcription \\
L & 122 & 2.9 & Replication, recombination and repair \\
B & 1 & 0 & Chromatin structure and dynamics \\
D & 25 & 0.6 & Cell cycle control, mitosis and meiosis \\
Y & 0 & 0 & Nuclear structure \\
V & 95 & 2.3 & Defense mechanisms \\
T & 138 & 3.3 & Signal transduction mechanisms \\
M & 166 & 3.9 & Cell wall/membrane biogenesis \\
N & 1 & 0 & Cell motility \\
Z & 0 & 0 & Cytoskeleton \\
W & 0 & 0 & Extracellular structures \\
U & 27 & 0.6 & Intracellular trafficking and secretion \\
O & 89 & 2.1 & Posttranslational modification, protein turnover, chaperones \\
\hline
\end{tabular}


Table 4. Number of genes associated with the 21 general COG functional categories

\begin{tabular}{lrrl}
\hline Code & Value & \multicolumn{1}{c}{$\%$} & Description \\
\hline J & 169 & 4 & Translation, ribosomal structure and biogenesis \\
G & 546 & 12.9 & Carbohydrate transport and metabolism \\
E & 264 & 6.3 & Amino acid transport and metabolism \\
F & 92 & 2.2 & Nucleotide transport and metabolism \\
H & 129 & 3.1 & Coenzyme transport and metabolism \\
I & 101 & 2.4 & Lipid transport and metabolism \\
P & 183 & 4.3 & Inorganic ion transport and metabolism \\
Q & 62 & 1.5 & Secondary metabolites biosynthesis, transport and catabolism \\
R & 433 & 10.3 & General function prediction only \\
S & 249 & 5.9 & Function unknown \\
- & 1116 & 26.4 & Not in COGs \\
\hline
\end{tabular}

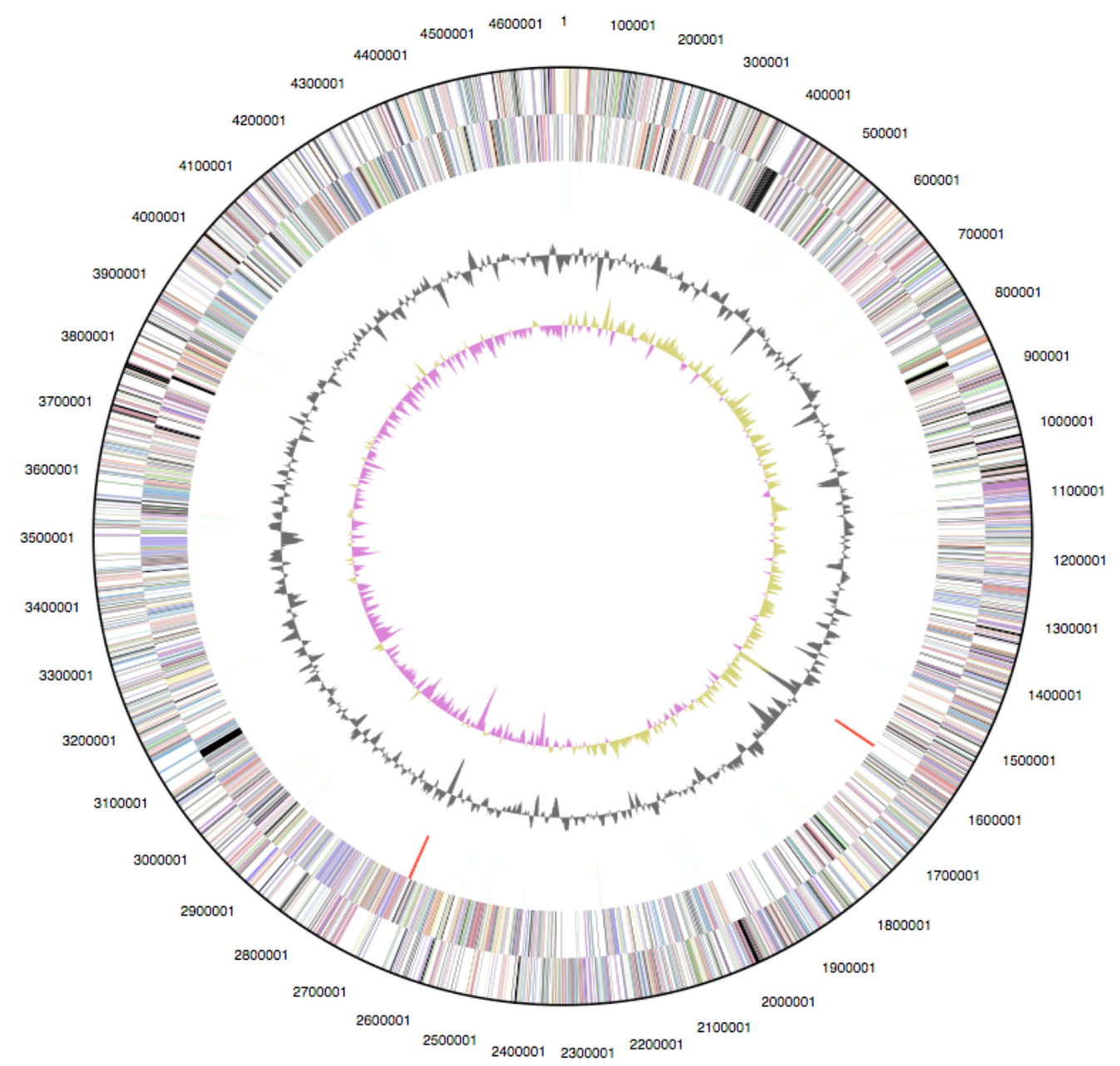

Figure 3. Graphical circular map of the genome. From outside to the center: Genes on forward strand (color by COG categories), Genes on reverse strand (color by COG categories), RNA genes (tRNAs green, rRNAs red, other RNAs black), GC content, GC skew 


\section{Acknowledgements}

We would like to gratefully acknowledge the help of Katja Steenblock for growing B. cavernae cultures and Susanne Schneider for DNA extraction and quality analysis (both at DSMZ). This work was performed under the auspices of the US Department of Energy's Office of Science, Biological and Environmental Research

\section{References}

1. Groth I, Schumann P, Schütze B, Augsten K, Kramer I, Stackebrandt E. Beutenbergia cavernae, gen. nov., sp. nov., a L-lysine-containing actinomycete isolated from a cave. Int / Syst Bacteriol 1999; 49:1733-40. PMID:10555355 doi:10.1099/00207713-49-4-1733

2. Zhi X-Y, Li W-J, Stackebrandt E. An update of the structure and 16S rRNA gene sequencebased definition of higher ranks of the class Actinobacteria, with the proposal of two new suborders and four new families and embedded descriptions of the existing higher taxa. Int J Syst Evol Micobiol 2009; 59:589-608. PMID:19244447 doi:10.1099/ijs.0.65780-0

3. von Wintzingerode F, Göbel UB, Siddiqui RA, Rösick $U$, Schumann P, Frühling A, Rohde M, Pukall R, Stackebrandt E. Salana multivorans gen. nov., sp. nov., a novel actinobacterium isolated from an anaerobic bioreactor and capable of selenate reduction. Int / Syst Evol Bacteriol 2001; 51:1653-61. PMID:11594592

4. Schumann, P, Kampfer, P, Busse, HJ, Evtushenko, LI Proposed minimal standards for describing new genera and species of the suborder Micrococcineae. Int I Syst Evol Microbiol. 2009 published ahead of print PMID: 19542112 doi:10.1099/ijs.0.12971-0

5. Lee C, Grasso C, Sharlow MF. Multiple sequence alignment using partial order graphs. Bioinformatics 2002; 18:452-64. PMID: $\underline{11934745}$ doi:10.1093/bioinformatics/18.3.452

6. Castresana J. Selection of conserved blocks from multiple alignments for their use in phylogenetic analysis. Mol Biol Evol 2000; 17:540-52. PMID:10742046

7. Stamatakis A, Hoover P, Rougemont J. A rapid bootstrap algorithm for the RAxML web-servers. Syst Biol 2008; 57:758-71. PMID:18853362 doi:10.1080/10635150802429642

8. Liolios K, Mavromatis K, Tavernarakis N, Kyrpides NC. The Genomes OnLine Database (GOLD) in 2007: status of genomic and metagenomic projects and their associated metadata.
Program, and by the University of California, Lawrence Berkeley National Laboratory under contract No. DEAC02-05CH11231, Lawrence Livermore National Laboratory under Contract No. DE-AC52-07NA27344, and Los Alamos National Laboratory under contract No. DEAC02-06NA25396, as well as German Research Foundation (DFG) INST 599/1-1.

Nucleic Acids Res 2008; 36:D475-9. PMID:17981842 doi:10.1093/nar/gkm884

9. Field D, Garrity G, Gray T, Morrison N, Selengut J, Sterk P, Tatusova T, Thomson N, Allen MJ, Angiuoli SV. Towards a richer description of our complete collection of genomes and metagenomes: the "Minimum Information about a Genome Sequence" (MIGS) specification. Nature Biotechnology 2008; 26:541-7. PMID:18464787 doi:10.1038/nbt1360

10. Stackebrandt E, Rainey FA, Ward-Rainey NL. Proposal for a new hierarchic classification system, Actinobacteria classis nov. Int J Syst Bacteriol 1997; 47:479-91. doi:10.1099/0020771347-2-479

11. Anonymous. Biological Agents: Technical rules for biological agents. <http://www.baua.de>.

12. Ashburner M, Ball CA, Blake JA, Botstein D, Butler H, Cherry JM, Davis AP, Dolinski K, Dwight SS, Eppig JT, et al. Gene ontology: tool for the unification of biology. The Gene Ontology Consortium. Nat Genet 2000; 25:25-29. PMID:10802651 doi:10.1038/75556

13. Yamada K, Komagata K. Taxonomic studies on coryneform bacteria IV. Morphological, cultural, biochemical, and physiological characteristics. J Gen Appl Microbiol 1997; 18:399-416.

14. Sims D, Brettin T, Detter JC, Han C, Lapidus A, et al. Complete genome of Kytococcus sedentarius type strain (strain 541T). SIGS, 2009 reviewed. doi: 10.4056/sigs.761

15. Anonymous. Prodigal Prokaryotic Dynamic Programming Genefinding Algorithm. Oak Ridge National Laboratory and University of Tennessee $2009<$ compbio.ornl.gov/prodigal/>>

16. Pati. GenePRIMP: A Gene Prediction Improvement Pipeline for microbial genomes. (Submitted).

17. Markowitz V, Mavromatis K, Ivanova N, Chen IM, Chu K, Palaniappan K, Szeto E, Anderson I, Lykidis A, Kyrpides N. Expert Review of Functional Annotations for Microbial Genomes. (Submited 2009). 